\section{Sur la Fabrication Electrochimique de l'acide azotique}

Dans le numéro de juin de la Revue, notre collaborateur M. P. Pierron, a exposé l'état actuel de l'Electrochimie des Nitrates (voir l'article : Deux débouchés de l'Energie hrdro-électrique). Dans cette étude il rappelait notamment qu'à la suite des recherches de lord Rayleigh et Ramsay, puis de Ramsay, Crookes calcula la dépense d'énergie correspondante à la formation d'un poids déterminé d'acide nitrique; un cheval, en 24 heures, produisait 36 gr. 75 , c'est-à-dire qu'avec le prix du cheval hydraulique au Niagara une tonne de nitrate de soude serait revenue à I 25 francs. Puis il décrivait les expériences récentes de Mac Dougall et Howles ayant obtenu un rendement maximum de $49 \mathrm{gr}$. 30 d'acide azotique par cheval -24 heures en opérant sur un mélange gazeux formé de deux volumes d'oxygène pour un volume d'azote et avec un courąnt de 0,3 ampères sous r roo volts. En admettant les bases du calcul de Crookes elles démontreraient la possibilité d'établir la torne de nitrate au prix de 1 o francs. Malheureusement ce ne sont encore là que des chiffres théoriques ainsi que le fait observer M. Pierron. Grâce à son obligeance, nous donnons aujourd'hui la traduction de l'intéressant article suivant que vient de publier la revue Iahrbuch der Elekrochemie, t. VIII, page 420, sur 1? procédé anglais Johnson. -

$$
\text { ** }
$$

La possibilité de combiner l'azote et l'oxygène dans l'étincelle électrique est de la plus haute importance pour le développement à venir des méthodes industriclles d'utilisation des réactions électrochimiques des gaz. Il est curieux et inexplicable que cette circonstance ait jusqu'ici peu éveillé l'attention. Les travaux de Crookes, Siemens et Halske, Losanitsch, Lord Rayleigh, Lepel, Salvadori, montrent la possibilité d'obtenir économiquement la précédente réaction grâce à cette circonstance que l'oxygène produit électrolytiquement peut être obtenu à assez bon marché pour qu'on puisse le mélanger à l'air de manière à réaliser la proportion $\mathrm{Az}^{2} \mathrm{O}^{3}$. De fait, les efforts se multiplient pour produire l'acide azotique ou azoteux par l'étincelle. Crookes, en 1897 , indiquait que 20.000 chevaux, qui coûtent au Niagara i 25 fr., donnent assez d'acide nitrique pour former une tonne dazotate de soude. Mac-Dougall et Howles ont obtenu de meilleurs résultats. Tandis que lord Rayleigh avec 12 chevaux obtenait $440 \mathrm{gr}$. d'acide, ils obtinrent, avec la même puissance, sur un mélange d'azote et d'oxygène dans la proportion de 1 à $2,590 \mathrm{gr}$. d'acide, c'est-à-dire que 20.000 chevaux donneraient environ ro tonnes d'acide. L'espoir que Swan base sur ce fait semble donc fondé; c'est la première fois que semble se justifier cette opinion que "les sources énormes d'énergie économique que sont les chutes d'eau du monde entier qui, à cette heure, se perdent sans résultat ", seront mises en valeur jusqu'aux points les plus reculés. Mais c'est une erreur que commet Kowalski lorsqu'il croit que l'on puisse produire en grand l'acide nitrique de cette manière par le courant d'une usine électrique urbaine en marche discontinue, pendant le temps où son énergie n'est pas utilisée à l'éclairage. Ce n'est pas dans cette voie qu'un ingénieur expérimenté s'attirera la confiance du public chimique.

Au sujet de la fabrique d'acile nitrique de Fribourg. (Suisse) qu'il cite et qui serait en construction (union de l'air et de vapeur d'eau par l'étincelle), rien n'a encore été publié.

Très remarquable est un procédé pour la production de combinaisons azotées à partir de l'azote atmosphérique de la "Atmospheric Products Comp. à Jersey City N.-Y. U. S. "Brev. Ang., No 823o (Igor) de Johnson. L'inventeur a établi :

$1^{\circ}$ Que le rendement est fortement influencé par le rapport de l'énergie de l'étincelle au volume de l'air traité. Son appareillage a pour but de travailler constamment avec le rapport reconnu une fois pour toutes comme le plus favorable.

$2^{\circ}$ Que la manière dont les étincelles se forment est également de grande importance pour le rendement. Ce ne sont ni la décharge silencieuse, ni la décharge ordinaire disruptive qui conviennent le mieux à la nitrification mais un arc et son efficacité dépend essentiellement, cela va sans dire, de sa nature même. Le plus. favorable est un arc aussi faible, c'est-à-dire aussi mince que possible, et lorsqu'il est sur le point de s'éteindre. Son action considérable est probablement due à la surface relativement grande effleuréc par l'air, par rapport à la quantité d'énergie transportée, circonstance qui éiève le rapport de l'effet synthétique à l'effet dissociant. Dans un arc de cette nature, l'intensité ne dépasse pas o,o I ampère à 0,00 I ampère. Comme pratiquement on ne peut demander le courant qu'à une dynamo, il faut produire le courant destiné à chaque arc par un transformateur spécial dont les dimensions sont choisies de manière que, la tension primaire étant fixée, l'intensité de l'arc ne puisse jamais dépasser une valeur déterminée d'avance. De plus, l'appareil est installé de telle façon que chaque arc s'éteigne de luimême neu de temps après son apparition et se rétablisse ensuite.

Les figures ci-jointes montrent schématiquement l'agencement des appareils. Par L (fig. I), l'air pénètre dans um récipient $G$ à parois résistant au feu. Dans celui-ci, l'étioncelle est produite par un appareil analogue au parafoudre à cornes de Siemens, inséré entre les deux extrémités du secondaire d'un transformateur $T$.

Tous ceux qui ont travaillé en laboratoire avec ce parafoudre si simple savent que, lorsque les deux fils en sont assez rapprochés en leurs points a, pour que l'étincelle: s'établisse, elle est aussitôt chassée vers le haut, puis s'épanouit et, finalement, s'éteint lorsque ses deux points de départ sont assez éloignés; enfin, elle se rétablit en a et le. phénomène recommence indéfiniment.

La figure 2 montre schématiquement le montage en parallèle de plusieurs de ces appareils, avec le dispositif d'absorption pour les gaz nitreux. Ceux-ci arrivent à travers le tube collecteur. $R$ dans une tour à acide sulfurique: 
$\mathrm{S}$, dans laquelle une pompe $\mathrm{P}$ déverse continuellement, par $\mathrm{E}$, de l'acide refroidi en $\mathrm{K}$ et puisé dans le récipient $\mathrm{V}$. De celui-ci, les oxydes de l'azote sont distillés par chauffage de l'acide et condensés en $\mathrm{C}$, d'où ils sortent en $\mathrm{F}$ où on les recueille.

Le montage des nombreux petits transformateurs nécessaires est coùteux et mal commode. L'inventeur indique pour cela une seconde disposition qui noffre rien de nouveau au point de vue électrique et nous paraît, si belle soit-elle estimée, moins propre que la première à assurer une marche sans à-coup. Nous renonçons à la reproduire.

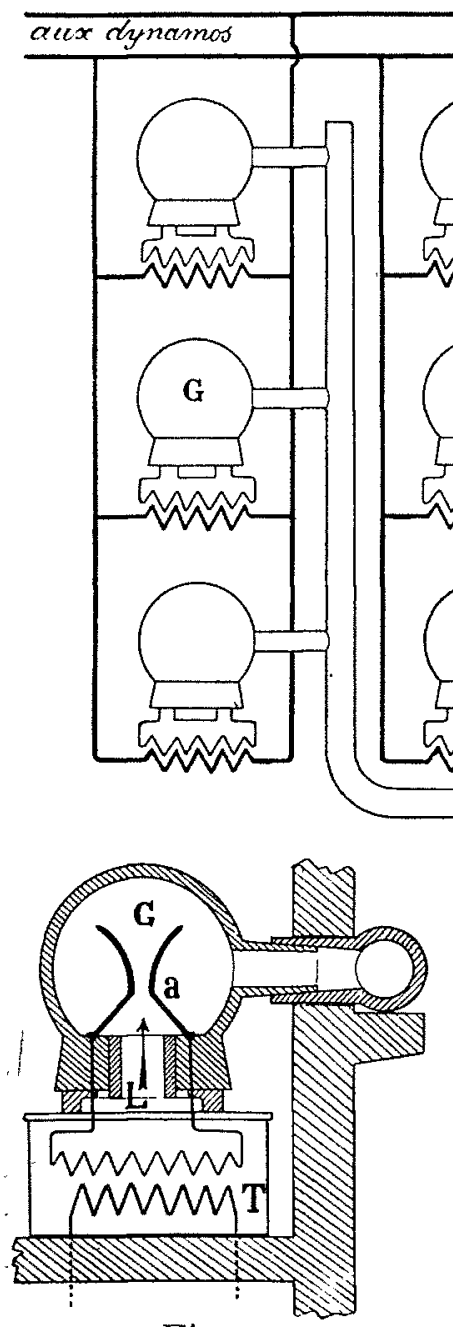

Fig. I

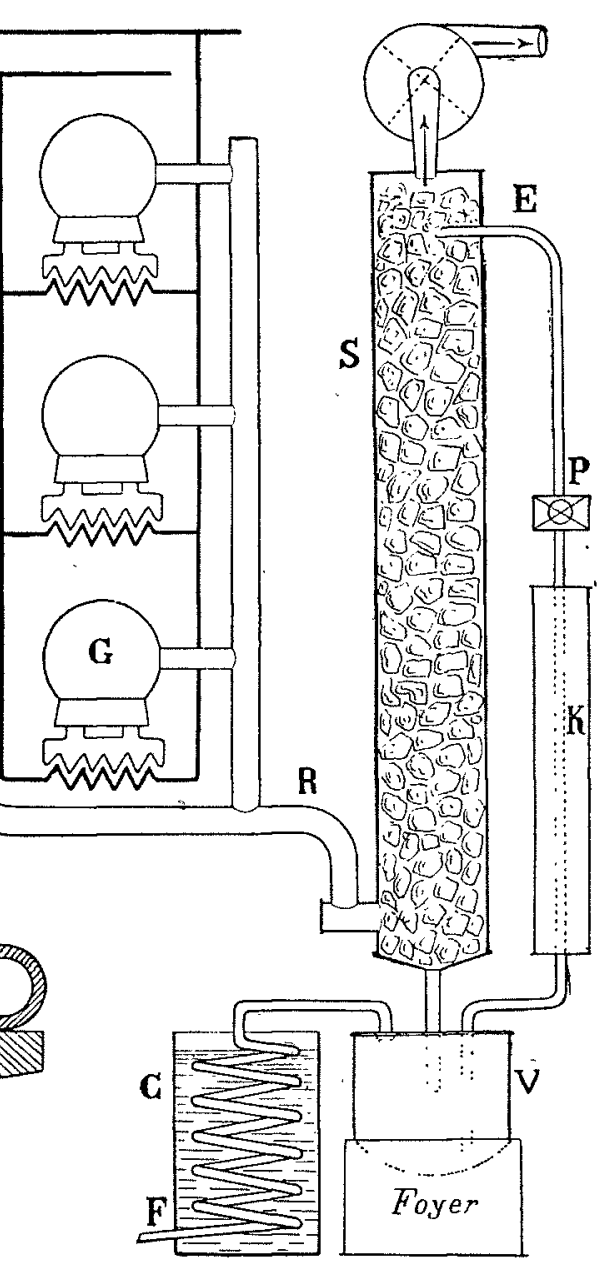

Fig. 2
La vitesse de l'air doit être réglée de manière à ne contenir que $3 \%$ de gaz nitreux. Les rendements sont alors les plus favorables.

Wilbert propose'les gaz des hauts fourneaux comme source d'énergie pour cette réaction.

Pour les raisons indiquées plus haut, les recherchés de Salvadori, purement chimiques (Chemiker Zeitung, I90 I-9), sur la combustion de l'air dans la flamme de l'hydrogène, intéresseront les électrochimistes.

$$
* *
$$

On remarquera que l'auteur n'indique pas le rendement de ce procéde qu'il qualifie lui-même de remarquable. Estce oubli ou faute de renseignements ? D'autre part, cette seçonde disposition électrique que l'inventeur indique corrme étant plus propre à assurer une marche sans àcoup et dont l'auteur semble faire peu de cas en renonçant à la décrire, peut être intéressante à signaler. Nous reviendrons sur ce sujet et tiendrons nos lecteurs au courant des essais si intéressants qui se poursuivent un peu de toute part sur la fabrication électrochimique des nitrates.

Il est - soit dit en passant - regrettable de voir l'étranger aller plus vite que nous dans cette voie, alors que notre industrie hydro-électrique est pourtant la première intéressée à la solution de ce problème d'une importance capitale pour son développement.

\section{LE MOIS HYDRO-ÉLECTRIQUE en France et à l'Etranger}

\section{INFORMATIONS DIVERSES}

\section{Une circulaire ministérielle sur les formalités néces- saires à l'autorisation des Barrages.}

Avant de reproduire le texte de cette circulaire, qui est rédigée dans des termes que l'on aurait pu désirer plus clairs, sans mềme se montrer trop exigeant, nous croyons devoir en donner l'analyse.

On sait que l'article 12 de la loi du 8 avril 1898 , dans son 3 e paragraphe, a annoncé un règlement d'administration publique pour déterminer " la forme de l'instruction qui doit précéder les arrêtés * des préfets relatifs à l'établissement, dans les rivières non navigables, a d'ouvrages intéressant le régime ou le mode de l'écoulement des " eaux ".Les autres ouvrages qui n'ont pas d'influence sur cet écoulement sont, en vertu de l'article ro de la même loi, dispensés de toute autorisation.

Le règlement annoncé n'a point encore paru; mais personne ne le réclame, car les demandes d'autorisation sont actuellement instruites dans les formes prescrites pour celles qui concernent les rivières classées, c'est-à-dire conformément à la circulaire, très complète et très claire,du 23 octobre I 851 . Toutefois le Ministre de l'Agriculture nous apprend que, le 2 t juin $\mathrm{i} \$ 99$, il a saisi le Conseil d'Etat de ce projet de règlement : La Haute Assemblée lui a répondu, que, pour elle, l'instruction faite dans les conditions ci-dessus indiquées est absolument suffisante, et que le besoin d'un nouveau texte ne se fait point sentir; qu'en tout cas ce règlement ne saurait être élaboré sans une étude préalable faite par les divers ministres compétents (Agriculture et Travaux Publics). Mais quelques chefs de service int, paraît-il, exprimé au ministre leurs inquiétudes au sujet de certaines autorisations demandées pour l'établissement de "barrages sans revanche ", c'est-à-dire sans la protection de oni 6 en contrebas des rives. Le besoin d'avoir une force puissante pousse les industriels à créer des barrages beaucoup plus importants que ceux qui, autrefois, étaient destinés à la mise en marche de minoteries ou de scieries. Que faire en pareil cas ? C'est à cette question que répond la circulaire.

Tout d'abord le ministre ne parait pas espérer que la solution soit contenue dans le règlement qui sera élaboré par la Commission interministériclle; le nouveau texte, dit-il, tiendra peut-être ces questions à l'écart, et confiera à l'administration la solution de chaque espèce possible. Dans ces conditions, le service de l'Hydraulique agricole et, après lui, le ministre, a pensé que, sans attendre la décision interministérielle, il est préférable de réserver à l'Administration SUPÉRIEURE - lisez : le service Hydraulique du Ministère de l'Agriculture - un droit de contrôle sur les établissements importants, d'oú il résulte que le préfet, tout en restant théoriquement maitre de l'autorisation définitive, ne devra la donner qu'après avis du ministre. 